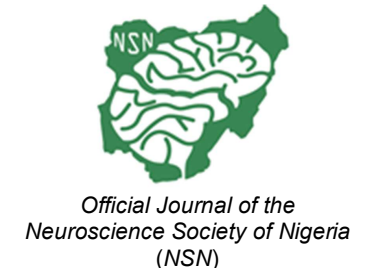
(NSN)

\title{
Nigella sativa Oil Preserved Anxiety-Like, Motor and Memory Related Behaviours and Neuronal Integrity in Dichlorvos Induced Acetyl Cholinesterase Inhibitions in Rats
}

\author{
Aminu Imam ${ }^{1}$, Christianah Oyegbola ${ }^{1}$, Maryam Busari ${ }^{1}$, Rukayat Jaji-Sulaimon ${ }^{1}$, \\ Abdulmusawwir Alli-Oluwafuyi ${ }^{2}$, Akeem A. Okesina ${ }^{3}$, Adam M. Afodun ${ }^{3,4}$, \\ Moyosore S. Ajao ${ }^{1}$
}

\author{
${ }^{1}$ Department of Anatomy, College of Health Sciences, University of Ilorin, Ilorin, Nigeria \\ ${ }^{2}$ Department of Pharmacology and Therapeutics, College of Health Sciences, University of Ilorin, Ilorin, Nigeria \\ ${ }^{3}$ Department of Anatomy, Faculty of Biomedical Sciences, Kampala International University, Uganda \\ ${ }^{4}$ Department of Anatomy and Cell Biology, Faculty of Health Sciences, Busitema University, Uganda
}

Received:

July 2021

Accepted:

October 2021

\begin{abstract}
Organophosphates are irreversible cholinesterase (ChE) inhibitors with neurological consequences, and there is not yet an effective antidote. Here, we investigated the effects of Nigella sativa oil (NSO) on the ChE inhibition, neurobehavioural and histopathological changes following dichlorvos (DDVP) ingestions in rats. Thirty-two male Wistar rats were randomised into four groups, receiving $1 \mathrm{ml} / \mathrm{kg}$ of normal saline, $8.8 \mathrm{mg} / \mathrm{kg}$ of DDVP, $8.8 \mathrm{mg} / \mathrm{kg}$ of DDVP and $1 \mathrm{ml} / \mathrm{kg}$ of NSO, and $1 \mathrm{ml} / \mathrm{kg}$ of NSO only respectively, for 14 consecutive days. Locomotor, anxiety-like behaviours and spatial working memory were assessed on the 14th day, using open field (OF), Y-maze and modified elevated plus maze paradigms. The rats were euthanized on the 15th day and the brains excised; three brains were fixed for histopathology, and the other five prepared for biochemical analysis of acetyl cholinesterase (AChE). DDVP exposure caused significant reductions in frontal, amygdala and cerebella AChE activity, spontaneous alternations, line crossing and rearing frequencies and time in centre square, and caused increase in freezing period, transfer latency and necrotic-like cells. NSO intervention was able to reverse DDVP effects on AChE activities, explorative, locomotor, anxiety and spatial memory behaviours in co-exposed rats. It also preserved the histological integrity of the investigated brain regions. It can be concluded that NSO, may be potent against organophosphates induced neurotoxicity and their neurobehavioural consequences through the modulation of AChE activities.
\end{abstract}

Key words: Nigella sativa; Dichlorvos; Acetyl cholinesterase; Neurobehavioural impairment; Neurodegeneration

\section{INTRODUCTION}

Pesticides are essential substances in agriculture and public health, as they repel, destroy and control pests (Richardson et al. 2019; Sidhu et al. 2019). The inevitable use of pesticides has led to increased exposures to humans (Robb and Baker 2018), which has been linked to various health burdens including respiratory, reproductive, nervous, hepatic, and renal abnormalities (Sidhu et al. 2019). The toxic effects of organochlorines, organophosphates and pyrethroid pesticides can affect non-target species like humans, because both pests and non-target species share similar molecular targets (Richardson et al. 2019). Organophosphates (OP) are mostly water soluble and disseminate easily into the environment through dissolution, abrasion and vocalization, causing

Correspondence: Aminu Imam, PhD, Department of Anatomy, Faculty of Basic Medical Sciences, University of Ilorin, P.M.B 1515, Ilorin, Nigeria. E-mail: imam.a@unilorin.edu.ng; Phone: +2348165663947; ORCID: 0000-0003-2371-3065 
undesirable effects in the biological systems (Sidhu et al. 2019). The principal mechanism of action for OPs is through the inhibition of acetyl cholinesterase (AChE), leading to a build-up of acetylcholine in synapses and hyperstimulation of cholinergic receptors in the central and peripheral nervous systems (Richardson et al. 2019). This massive buildup of acetylcholine due to OP intoxication has been observed in various neurological disorders with deleterious effects like convulsions, oxidative damage, impaired neuropsychological performance and neurobehavioural deficits, including depression, anxiety and stress amongst others (Imam et al. 2018a; Guignet and Lein 2019).

Nigella sativa (NS), also identified as black seed or
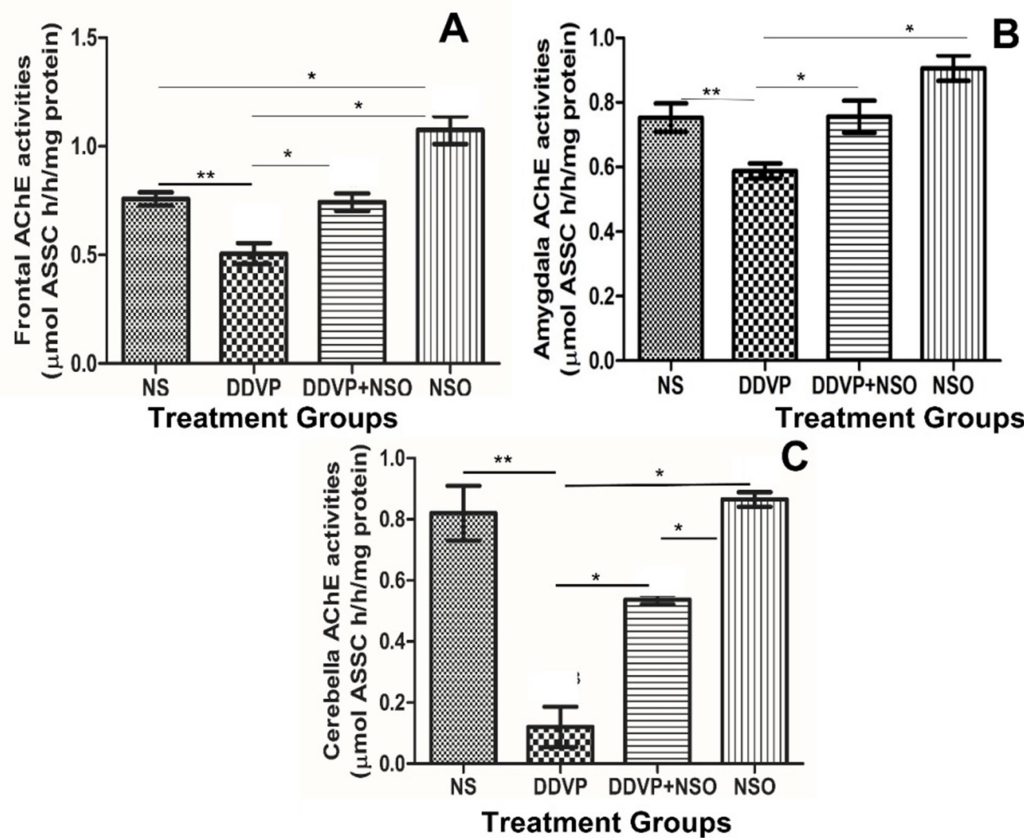

Fig. 1: Frontal (A), amygdala (B) and cerebellar (C) AChE activities in rats exposed to normal saline (NS), dichlorvos (DDVP), dichlorvos + Nigella sativa Oil (DDVP+NSO) and NSO only. ${ }^{* *}$ - Significant $(p \leq 0.05)$ compared with the control, ${ }^{*}$ - Significant $(p \leq 0.05)$ compared to the DDVP group and/or the control $(n=5)$.

black cumin, is a flowering plant belonging to the Family, Ranunculaceae (Cascella et al. 2018). NS is a medicinal plant that has been used over the years in the management of various diseases (Islam et al. 2019; Yimer et al. 2019; Begum and Mannan 2020; Pop et al. 2020). Phytochemicals of NS offer protection against toxicity caused by essential used and accidentally leaked environmental chemicals (Assi 2019). The NS plant is rich in fixed oils (mainly fatty acids $-30 \%)$, volatile oils $(0.40-0.45 \%)$, vitamins, amino acids, proteins, carbohydrates, alkaloids, saponins, crude fibre and minerals (Cascella et al. 2018). It also contain in abundance, polyunsaturated fatty acids, phytosterols, thymoquinone (TQ), carvacrol and t-anethole among others, of which thymoquinone is considered as the primary active agent.

The combined phytochemical constituents of NS afford it a protective and curative remedy for many disorders (Cascella et al. 2018), specifically as an antioxidant, anti-inflammatory, antidiabetic, anti-cancer, antidepressant, immunomodulatory, analgesic, memory enhancing, neuroprotective agent in idiopathic and chemical induced toxicity (including OPs), as well as reactivating AChE activities and being efficacious against neurodegenerative diseases (Ikhsan et al. 2018; Alhibshi et al. 2019; Fanoudi et al. 2019; Bordoni et al. 2019; Norouzi et al. 2019; Ahirwar and Ahirwar 2020; Asrar et al. 2020; Begum and Mannan 2020).

This study investigated the ameliorative effect of Nigella sativa oil (NSO) against dichlorvos (DDVP) induced acetyl cholinesterase inhibition, neurobehavioural impairment, and neurodegenerative-like effects in rats.

\section{MATERIALS AND METHODS}

\section{Ethical Approval}

This research work was approved by the University of Ilorin Ethical Review Committee (UERC) (UERC/ASN/2017/ 856), following the recommendation of the College of Health Sciences ethical review committee, in compliance with the Institutional Animal Care and Use Committee (IACUC).

\section{Chemicals and Drugs}

The dichlorvos solution (PRIMEFORC$\mathrm{E}$, Anhui Zhongshan Chemical Industry Co Ltd, China) obtained at a local agrochemical store in Ilorin, Nigeria. A portion of the solvent was then dissolved in normal saline to give a dose of $14.9 \mathrm{mg} / \mathrm{kg}$.bw.

The Nigella sativa oil (HUSNA, Fazhab Agency, Karachi, Pakistan) concentration; 100\% black seed) was obtained from a local store in Ilorin, Nigeria.

\section{Animal Care}

Thirty-two adult male Wistar rats weighing between 150 and $170 \mathrm{~g}$ were obtained from the University of llorin Biological garden, llorin. They were housed in cages and fed with standard laboratory diet and water ad libitum in the animal holding unit of the Faculty of Basic Medical Sciences, College of Health Sciences, University of Ilorin, llorin. The rats were exposed to a $12 \mathrm{~h}$ light/dark cycle at room temperature for 7 days before the commencement of the experiment. All rats were handled in accordance 
with the standard guide for the care and use of laboratory animals.

\section{Experimental Design}

The rats were randomly divided into four groups $(n=8)$ as follows: Group 1 (control) received normal saline $(1 \mathrm{ml} / \mathrm{kg})$, group 2 received DDVP $(8.8 \mathrm{mg} / \mathrm{kg})$, group 3 received DDVP $(8.8 \mathrm{mg} / \mathrm{kg})$ and NSO (1 $\mathrm{ml} / \mathrm{kg}$ ), while group 4 received NSO (1 $\mathrm{ml} / \mathrm{kg}$ ). Administrations were orally, and daily for 14 days. All procedures were scheduled and carried out during the early phase of the day between 07:00 and 09:00 hours.
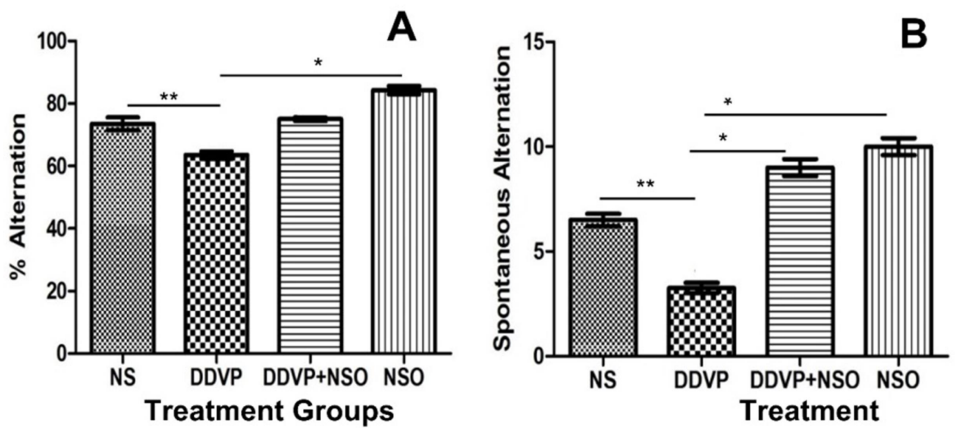

Fig. 2: Percentage (A) and spontaneous (B) alternations as measures of spatial working memory of rats exposed to normal saline (NS), dichlorvos (DDVP), dichlorvos + Nigella sativa Oil (DDVP+NSO) and NSO only (NSO). ${ }^{* *}$ - Significant $(p \leq 0.05)$ compared with the control; * Significant $(p \leq 0.05)$ compared to the DDVP group $(n=5)$.

\section{Behavioural Evaluation \\ Y-Maze Test}

Spontaneous and percentage alternations were analysed using symmetrical Y-maze under reduced light conditions. The Y-maze apparatus was made of three wooden arms, joined together at the middle, forming a "Y" shape. The arms were labelled A, B and $C$ in an anti-clockwise rotation and the rats were trained to enter each arm following the letters consecutively, thereby allowing the rats to make only right turns within the maze. In the recorded test, rats were subjected to the maze for 5 min each with all
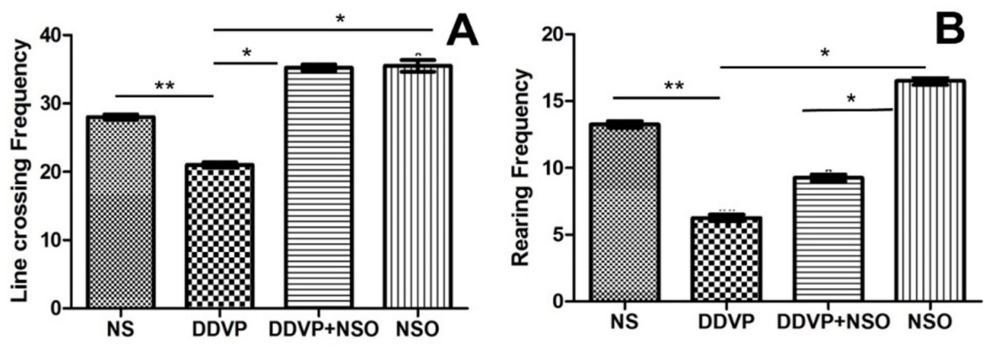

Fig. 3: Line crossing (A) and rearing frequencies (B) as measures of exploratory activities of rats exposed to normal saline (NS), dichlorvos (DDVP), dichlorvos + Nigella sativa oil (DDVP+NSO) and NSO only. ${ }^{* *}$ - Significant $(p \leq 0.05)$ compared with the control; * Significant $(p \leq 0.05)$ compared to the DDVP exposed rats and/or other groups $(n=5)$.

\section{paradigm. This} surrounded by a short edge to prevent falls, and two enclosed arms erected in such a way that the two open arms were opposite each other. The maze was raised about $35 \mathrm{~cm}$ above the ground with a table stand and the arms of the maze were connected by a central platform. At each of the two trials, each rat was gently placed on an open arm, positioned to face away from the central platform and the closed arms. The time it took the rats to recognise the threat and move to the closed arms was recorded as the transfer latency. While the first trial was for acquisition; the second was used as a measure of B fear learning.

\section{Biochemical Evaluation}

At the end of the treatment period, the animals were anaesthetized, sacrificed, and the brains were quickly removed and weighed. The brains were sectioned and the frontal cortices, amygdala and cerebellum portions were removed. Since acetyl cholinesterase inhibition is the pathological hallmark of organophosphate poisoning, AChE levels are frequently used for the diagnosis of organophosphate exposure. The homogenized tissues were placed in phosphate buffer with Triton-X 1 and centrifuged at $7800 \mathrm{~g}$ for $5 \mathrm{~min}$ at 4 
${ }^{\circ} \mathrm{C}$. The following reagents were used; $35 \mu \mathrm{L}$ of $5 \mathrm{mM}$ dithio-bisnitrobenzoic acid (DTNB), $10 \mu \mathrm{L}$ of $75 \mathrm{mM}$ acetylthiocholine (ATCh) and $820 \mu \mathrm{L}$ of $0.1 \mathrm{M}$ phosphate buffer ( $\mathrm{pH}$ 8.0). Protein concentration in the tissue homogenates were quantified using a Bradford assay and AChE activity was calculated in micromoles of ATCh hydrolysed per hour per milligram of protein. AChE activity was expressed as percentage of control activity and measured values in micromole per hour per milligram of protein.

\section{Histological Evaluations}

The other portions of the frontal and cerebella tissues were fixed in $10 \%$ neutral buffered formalin for $24 \mathrm{~h}$ and embedded in paraffin. Sections of $5 \mu \mathrm{m}$ thickness were prepared, attached to slides, deparaffinised and stained with cresyl fast violet (CFV) for general cyto-architectural examination.

\section{Statistical Analysis}

Data from the behaviour and biochemical tests were analysed using One-way analysis of variance (ANOVA), and subjected to post hoc Bonferroni's multiple comparison test. The results are expressed as mean \pm SEM. Statistical analyses were performed using Graph Pad Prism software (version 5.0, La Jolla, CA). Values of $\mathrm{p} \leq 0.05$ were considered statistically significant. significantly $(p \leq 0.05)$ increased these parameters compared to the DDVP treated group (Fig. 2 A and B).

Nigella sativa Oil Improved the Impaired
Explorative Behaviours Caused by DDVP
Exposure
Exposure to DDVP significantly $(p \leq 0.05)$ reduced line crossing and rearing frequencies when compared with the control, while treatment with NSO significantly $(p \leq 0.05)$ increased these parameters compared to the DDVP treated group (Figure $3 \mathrm{~A}$ and B).

Effects of DDVP and NSO on Anxiety-like and Related Behaviours and Learning

Exposure to DDVP significantly $(p \leq 0.05)$ increased anxiety-like behaviour, marked by a reduction in time in centre square (Fig. 4A), increase in freezing period (Fig. 4B) and increase in transfer latency (Fig. 4C) when compared with the control, while treatment with NSO significantly $(p \leq 0.05)$ reversed these parameters when compared to the DDVP treated group (Fig. 4 A and B).

\section{Nigella sativa Oil Preserved the Frontal and Cerebellar Histo-architecture Following DDVP Exposure}

Some marked histo-architectural deteriorations, like vacuolation, where observed in the frontal and cerebella brain regions of Wistar rats following exposure to DDVP. These effects were however,

Nigella sativa Oil Increased Acetyl Cholinesterase Activity Following AChE Level Depletion Induced by DDVP Exposure

Dichlorvos was observed to be an acetyl cholinesterase (AChE) inhibitor as AChE activity was significantly lower $(p \leq 0.05)$ in the frontal, amygdala, and cerebella brain regions, compared to that of the control group, following exposure to DDVP, with marked depletion in the cerebellum (Fig. 1). NSO however, increased AChE activity across these brain regions in the animal groups that received both DDVP and NSO concurrently. The animal group that received NSO alone also had higher levels of AChE compared to the group that received DDVP alone, and to the control group (Fig. 1).

Nigella sativa Oil Improved the Impaired Spatial Working Memory caused by DDVP Exposure

Exposure to DDVP significantly ( $p \leq 0.05$ ) reduced \% alternation and spontaneous alternation when compared with the control, while treatment with NSO
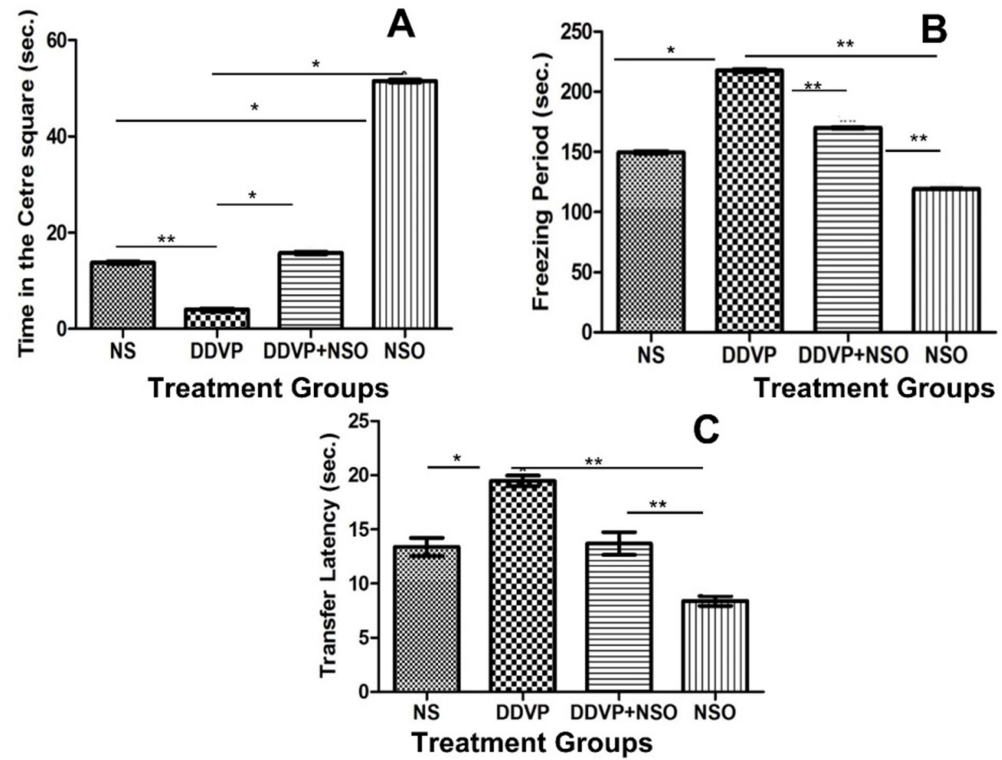

Fig. 4: Time in centre square (A), freezing period $(B)$ and transfer latency $(C)$ as measures of anxiety-like behaviours in rats exposed to normal saline (NS), dichlorvos (DDVP), dichlorvos + Nigella sativa oil (DDVP+NSO) and NSO only. ** -Significant $(p \leq 0.05)$ compared with the control or DDVP group; * - Significant $(p \leq 0.05)$ compared to the DDVP and/or control group $(n=5)$. 
absent in the experimental groups that received DDVP and NSO concurrently (Fig. 5 and 6). NSO was observed to protect the cortices significantly and reduced the severity of the degenerative-like effects of DDVP.

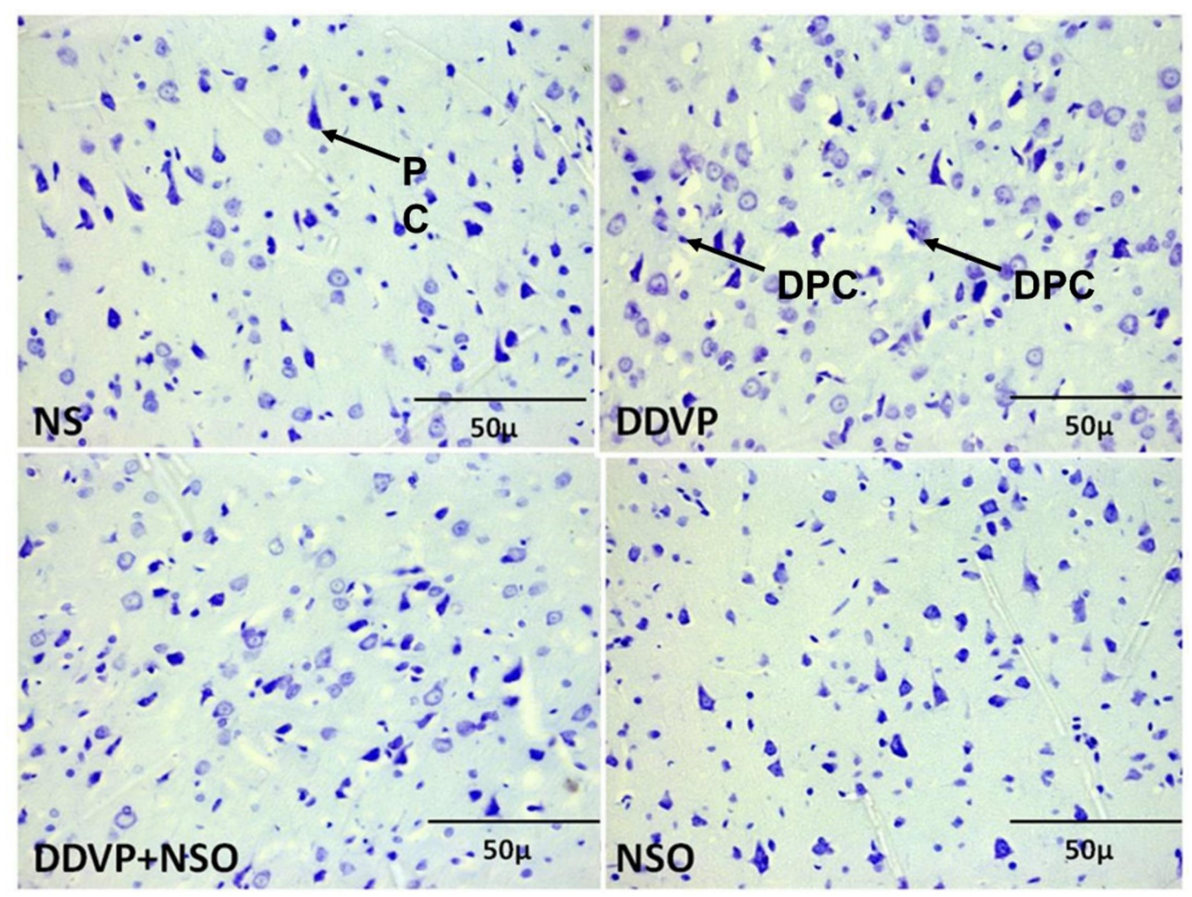

Fig. 5: Representative photomicrographs of frontal cortices of rats exposed to Normal saline (NS), Dichlorvos (DDVP), Dichlorvos + Nigella sativa oil (DDVP+NSO) and NSO. PC - pyramidal cell, DPC -degenerating pyramidal cell. CFV $100 \times$.

\section{DISCUSSION}

Organophosphate pesticide (OP) exposure, including dichlorvos may occur through inhalation, ingestion or dermal contact (Richardson et al. 2019). They exert their toxic effects by inhibiting the activity of acetyl cholinesterase at the synapses and neuromuscular junctions, through the phosphorylation of the serine residue within the active site of the enzyme, thus blocking the degradation of the neurotransmitter acetylcholine (ACh) (Franjesevic et al. 2019; AbdelDaim et al. 2020), thus are generally characterised by cholinergic toxicity, neurobehavioral, molecular, and enzymatic effects (Blotnick-Rubin and Anglister 2018; Ezz El-Din et al. 2019; Schmitt et al. 2019).

The current study investigated the efficacy of Nigella sativa in DDVP influence on the activity of acetyl cholinesterase in selected brain regions, and how this may be related to some behaviour parameters in the exposed rats or culminate in histoarchitectural changes in the brain regions (frontal and cerebella cortices). In this study, DDVP successfully inhibited AChE activity as expected and reported in some of our previous studies on AChE activities following exposures to organophosphates, including diclorvos and chlorpyrifos (Imam et al. 2018 a,b,c).

However, administration of NSO was observed to reactivate the AChE activity in the amygdala, frontal cortex and cerebellum. The reactivation of $\mathrm{AChE}$ activity by NSO may be attributed to its ability to act like an oxime by cleaving the $\mathrm{P}-\mathrm{O}($ Ser) bond that was formed during the initial inhibition (characterised by the formation of a covalent P-O(Ser) bond between the OP and catalytic serine of AChE) (Franjesevic et al. 2019).

Noting the previous reports that OPs inhibition of AChE activities will not only lead to overstimulation of cholinergic receptors, causing cholinergic toxicity (Blotnick-Rubin and Anglister 2018; Ezz El-Din et al. 2019), but have also been implicated in predisposing neurodevelopmental disorder$s$ and neurodegenerative diseases (Jokanović 2018; Guignet and Lein 2019; Tang 2020). A variety of possible behavioural effects of the induced cholinergic toxicity, including locomotor, anxietylike, anxiety related learning and spatial working memory were evaluated.

Repeated oral dichlorvos exposure in this study prevented the exposed rats from exploring the open field paradigm, evidenced in the reduced rearing and line crossing frequencies, and the time spent in centre square, suggestive of combined impaired locomotor behaviours and possible anxiety (Belovicova et al. 2017). Strengthening our conclusion of DDVP induced anxiety-like behaviours, was the observed delay in transfer latency in mEPM paradigm, which is a measure of anxiety related leaning.

Cholinergic toxicity, impaired motor and anxiety behaviours are important factors in neurological disorders or neurotoxicity induced memory impairments, thus the impaired spatial working memory and the fear related learning in the dichlorvos exposed rats are not unrelated. According to Ansari et al. (2019) the use of AChE inhibitor such as organophosphates, is also one of the most important strategies for memory impairment, and exposures to various environmental toxins are associated with the pathogenesis of neurological dysfunctions (Imam et al. 2018a,b,c; Suarez-Lopez et al. 2019). 
Consequently, the coadministration with Nigella sativa has not only been reported to improve cholinergic functions as reported in this study and in the literature (Fanoudi et al. 2019; Imam et al. 2019). Our present result is similar to the reports of improved spatial memory and other memory indices (Imam et al. 2018 a,b,c; Fanoudi et al. 2019; Norouzi et al. 2019; Asrar et al. 2020), improved motor coordination (Folarin et al. 2020), exploratory and locomotor behaviours (Imam et al. 2016) and improved anxiety or depressive-like behaviours (Ahirwar and Ahirwar 2020) in toxicity models and neurodegenerative diseases (Cascella et al. 2018; Samarghandian et al. 2018).

The therapeutic efficacy of Nigella sativa in improving neuro-cognitive behaviours neurotoxicity, modelled neurodegeneration and in this study may also be related to its antioxidant, anti-inflammatory (Begum and Mannan 2020) and neuroprotective (Isaev et al. 2020) effects. Exposure to organophosphates is considered a risk factor for many neurodegenerative diseases (Guignet and Lein 2019). Complementing the induced cholinergic toxicity and impaired neurobehavioural activities described above, is the observed degenerative-like effects of dichlorvos on the cellular integrities of the frontal and cerebella brain regions. And the oral interventions with the oil of Nigella sativa sufficiently protected the brain regions. These findings are not unrelated to the reported cytoprotective or histoprotective activity of NSO or its active ingredient, thymoquinone in induced pluripotent stem cell-derived cholinergic neurons (Alhibshi et al. 2019), human pre-adipocytes (Bordoni et al. 2019) and cerebellum of hypopersed rats (Fanoudi et al. 2019). More specifically, it was reported to be neuroprotective, preserving the cytoarchitectural integrity of different brain regions in organophosphate toxicity (Imam et al. 2018a, b, c; Imam et al. 2019). Our study result confirms the neuroprotective effect of Nigella sativa against neurodegeneration.

\section{Conclusion}

Nigella sativa preserved the DDDVP impaired ChE activity, locomotor activity, anxiety-like behaviours, memory indices, and neuronal integrities in the exposed rats. Thus, Nigella sativa may be a potent natural agent in the treatment of organophosphate neurotoxicity.

\section{Grants and financial support}

Nil.

\section{Conflict of Interest}

None declared.

\section{Acknowledgements}

We acknowledge the Ilorin Neuroscience Group for providing a subsidized behavioural paradigms for training and experimentation by members and the University of llorin for the consumables used for the histological processes and staining.

\section{Authors Contribution}

Al - Conceptualization; AI, CO, MB, RJ-S and AA-O Methodology; Al, AMA and MSA - validation; Al, AAO, RJ-S, MB and AA-O - formal analysis; Al, AA-O and MSA - resources; Al, RJ-S, AAO, MB and AMA data curation; $\mathrm{Al}, \mathrm{CO}$ and $\mathrm{MB}$ - writing-original draft preparation, AI, RJ-S, AA-O, AAO, AMA and MSA writing-review and editing; AMA and MSA - 
visualization; Al and MSA - supervision; Al and MSA - project administration.

\section{REFERENCES}

Abdel-Daim, M.M., Abushouk, A.I., Bungău, S.G., Bin-Jumah, M., El-Kott, A.F., Shati, A.A., et al. (2020) Protective effects of thymoquinone and diallyl sulphide against malathion-induced toxicity in rats. Environ Sci Pollut Res. 27(10):10228-10235.

Ahirwar, D. and Ahirwar, B. (2020) Antidepressant effect of Nigella sativa in stress-induced depression. Res J Pharm Technol. 13(4):1611-1614.

Alhibshi, A.H., Odawara, A. and Suzuki, I. (2019) Neuroprotective efficacy of thymoquinone against amyloid beta-induced neurotoxicity in human induced pluripotent stem cell-derived cholinergic neurons. Biochem Biophys Rep. 17:122-126.

Ansari, M., Mandegary, A., Mosalanejhad, N., Asadi, A. and Sharififar, F. (2019) Nigella sativa L., supplementary plant with anticholinesterase effect for cognition problems: A kinetic study. Curr Aging Sci. 13(2):129-135.

Asrar, B., Khan, I. U., Homberg, J. R. and Haleem, D. J. (2020) Nigella sativa oil ameliorates chronic ethanol induced anxiety and impaired spatial memory by modulating noradrenaline levels. Pak Vet J. 40(3): 350-354.

Assi, M.A. (2019) Reversal of hemato-biochemical alterations induced by lead acetate treatments with Eurycoma longifolia and Nigella sativa in rats. Plant Arch. 19(2):920-926.

Begum, S. and Mannan, A. (2020) A review on Nigella sativa: a marvel herb. J Drug Deliv Ther. 10(2):213-219.

Belovicova, K., Bogi, E., Csatlosova, K. and Dubovicky, M. (2017). Animal tests for anxiety-like and depression-like behavior in rats. Interdiscip Toxicol. 10(1):40-43.

Blotnick-Rubin, E. and Anglister, L. (2018) Fine localization of acetylcholinesterase in the synaptic cleft of the vertebrate neuromuscular junction. Front Mol Neurosci. 11:123.

Bordoni, L., Fedeli, D., Nasuti, C., Maggi, F., Papa, F., Wabitsch, M., et al. (2019) Antioxidant and antiinflammatory properties of Nigella sativa oil in human pre-adipocytes. Antioxidants. 8(2):51.

Cascella, M., Bimonte, S., Barbieri, A., Del Vecchio, V., Muzio, M. R., Vitale, A., et al. (2018) Dissecting the potential roles of Nigella sativa and its constituent thymoquinone on the prevention and on the progression of Alzheimer's disease. Front Aging Neurosci. 10:16.

Ezz El-Din, E. M., Osman, K. A., Ahmed, N. S. and El-Seedy, A. S. (2019) Adverse outcomes of repeated chlorpyrifos-ethyl and methyl exposure in rats: the ameliorating role of $\mathrm{N}$-acetylcysteine. ASEJ. 40:618-628.
Fanoudi, S., Alavi, M.S., Hosseini, M. and Sadeghnia, H.R. (2019) Nigella sativa and thymoquinone attenuate oxidative stress and cognitive impairment following cerebral hypoperfusion in rats. Metab Brain Dis. 34(4):1001-1010.

Folarin, R.O., Surajudeen, O.B., Omotosho, E.O., Owoniyi, A.O., Oyeleye, D.O. and Shallie, P. (2020) Motor co-ordinative roles of Nigella sativa oil in mice models of phenol-induced essential tremor. Ann Health Res. 6(1):85-99.

Franjesevic, A.J., Sillart, S.B., Beck, J.M., Vyas, S., Callam, C.S. and Hadad, C.M. (2019) Resurrection and reactivation of acetylcholinesterase and butyrylcholinesterase. Chem Eur J. 25(21):53375371.

Guignet, M. and Lein, P. (2019) Neuroinflammation in organophosphate induced neurotoxicity. Adv Neurotox. 3:35-79

Ikhsan, M., Hiedayati, N., Maeyama, K. and Nurwidya, F. (2018) Nigella sativa as an antiinflammatory agent in asthma. BMC Res Notes. 11: 744

Imam, A., Adebayo, M., Abdulmajeed, W. I., AlliOluwafuyi, A., Amin, A., Ibrahim, A., Gwadabe, S., et al. (2018a) Dichlorvos induced AChE inhibition in discrete brain regions and the neuro-cognitive implications: ameliorative effect of Nigella sativa. Iran J Toxicol. 12(5):11-16.

Imam, A., Ogunniyi, A., Ibrahim, A., Abdulmajeed, W.I., Oyewole, L.A., Lawan, A.H., et al. (2018b) Dichlorvos induced oxidative and neuronal responses in rats: mitigative efficacy of Nigella sativa (black cumin). Nig J Physiol Sci. 33(1) 83-88.

Imam, A., Teslimat, A.J., Victoria, W., Samson, C., Aboyeji, O.L., Olatunbosun, O., et al. (2019) Nigella sativa oil protected the hippocampus against acetyl cholinesterase and oxidative dysfunctions-driven impaired working memory in rats. Bull Fac Pharm Cairo Univ. 57(1):25-34.

Imam, A., Alakoso, T., Williams, V., Chengetanai, S., Oyewole, L.A., Olaleye, O., et al. (2018c) Nigella sativaoil protected the hippocampus against acetylcholinesterase (AChE) and oxidative dysfunction-driven impaired working memory in rats. Bull Fac Pharm Cairo Univ. 5(1):25-34.

Imam, A., Ajao, M.S., Abdulbasit, A., Abdulmajeed, W.I., Ibrahim, A., Olajide, O.J. et al. (2016) Cannabisinduced moto-cognitive dysfunction in Wistar rats: ameliorative efficacy of Nigella sativa. Malays $\mathrm{J}$ Med Sci. 23(5):17-28.

Isaev, N.K., Chetverikov, N.S., Stelmashook, E.V., Genrikhs, E.E., Khaspekov, L.G. and Illarioshkin, S.N. (2020) Thymoquinone as a potential neuroprotector in acute and chronic forms of cerebral pathology. Biochemistry (Moscow). 85(2):167-176.

Islam, M.T., Khan, M.R., and Mishra, S.K. (2019) An updated literature-based review: phytochemistry, pharmacology and therapeutic promises of Nigella sativa L. Orient Pharm Exp Med. 19(2):115-129. 
Jokanović, M. (2018) Neurotoxic effects of organophosphorus pesticides and possible association with neurodegenerative diseases in man: A review. Toxicol. 410:125-131.

Norouzi, F., Hosseini, M., Abareshi, A., Beheshti, F., Khazaei, M., Shafei, M.N., et al. (2019) Memory enhancing effect of Nigella Sativa hydro-alcoholic extract on lipopolysaccharide-induced memory impairment in rats. Drug and chemical toxicology, 42(3):270-279.

Pop, R., Trifa, A., Popolo, A., Chedea, V., Militaru, C., Bocsan, I, et al. (2020) Nigella sativa: Valuable perspective in the management of chronic diseases. Iran J Basic Med Sci. 23(6):699-713.

Richardson, J.R., Fitsanakis, V., Westerink, R.H. and Kanthasamy, A.G. (2019). Neurotoxicity of pesticides. Acta Neuropathol. 138(3):343-362.

Robb, E.L. and Baker, M.B. (2018) Organophosphate toxicity. In: Stat Pearls [Internet]. Stat Pearls Publishing.

Samarghandian, S., Farkhondeh, T. and Samini, F. (2018) A review on possible therapeutic effect of Nigella sativa and thymoquinone in neurodegenerative diseases. CNS Neurol Disord Drug Targets. 17(6):412-420.
Schmitt, C., McManus, M., Kumar, N., Awoyemi, O. and Crago, J. (2019) Comparative analyses of the neurobehavioral, molecular, and enzymatic effects of organophosphates on embryo-larval zebrafish (Danio rerio). Neurotoxicol Teratol. 73:67-75.

Sidhu, G. K., Singh, S., Kumar, V., Dhanjal, D. S., Datta, S. and Singh, J. (2019) Toxicity, monitoring and biodegradation of organophosphate pesticides: a review. Crit Rev Environ Sci Technol. 49(13):11351187.

Suarez-Lopez, J., Suarez-Torres, J., Sheila, G., Lopez-Paredes, D. and Noble, M. (2019) Acetylcholinesterase inhibition and symptoms of depression and anxiety among adolescents in agricultural communities in Ecuador: transient effects on mood. Environ Epidemiol. 3:386

Tang, B.L. (2020) Neuropathological mechanisms associated with pesticides in Alzheimer's disease. Toxics. 8(2):21.

Yimer, E.M., Tuem, K.B., Karim, A., Ur-Rehman, N. and Anwar, F. (2019) Nigella sativa L. (black cumin): a promising natural remedy for wide range of illnesses. Evid Based Complementary Altern Med. 2019:1528635. https://doi.org/10.1155/2019/1528635

Cite as: Imam, A., Oyegbola, C., Busari, M., Jaji-Sulaimon, R., Alli-Oluwafuyi, A., Okesina, A. A., Afodun, A. M. and Ajao, M. S. (2021). Nigella sativa oil preserved anxiety-like, motor and memory related behaviours and neuronal integrity in dichlorvos induced acetyl cholinesterase inhibitions in rats. Nig. J. Neurosci. 12(3):84-91. http://doi.org/10.47081/njn2021.12.3/002 